\title{
A Control System Design to Establish Dose-Response Relationships in Wound Healing Therapy
}

\author{
Jacquelyn Dawn Parente, Knut Möller \\ Institute of Technical Medicine, Furtwangen University, Villingen-Schwenningen, Germany \\ Email: pjd@hs-furtwangen.de
}

How to cite this paper: Parente, J.D. and Möller, K. (2017) A Control System Design to Establish Dose-Response Relationships in Wound Healing Therapy. J. Biomedical Science and Engineering, 10, 76-85. https://doi.org/10.4236/jbise.2017.105B009

Received: April 13, 2017

Accepted: May 3, 2017

Published: May 10, 2017

\begin{abstract}
Advanced biophysical wound healing therapies can apply mechanical, electrical, or light energy to re-stimulate healing processes in chronic wounds. Despite the growing evidence of the clinical efficacy of these therapies, the optimal treatment stimulation parameters remain unknown and there are no standard treatment protocols. We introduce a closed-loop control design as an experimental system to study the dose-response of wound healing therapy treatment within a prescribed multidimensional and multimodal stimulation parameter space. Systems engineering approaches are applied to the control problem for estimation of a transfer function and model equations derived for use in optimal model-based control. The experimental control system design consisted of simultaneous application of biophysical energies inputted into a wound system. A study design set up including the use of negative pressure wound therapy, electrical stimulation therapy, and photobiomodulation device systems was described. Treatment stimulation parameters were selected from experimental ranges used in the scientific literature. Classical control methods and model-based control were suggested for model selection and evaluation and design of the overall control system. An experimental design for multimodal biophysical wound healing therapy control system is introduced to establish the dose-response interactions for development of therapeutic applications and device design.
\end{abstract}

\section{Keywords}

Chronic wounds, High voltage pulsed current, Low level light therapy, Negative pressure wound therapy, Modeling and control, Photobiomodulation, Wound healing

\section{Introduction}

In Germany during 2012, around 197000 new patients were added to the grow- 
ing number of 786,000 patients living with chronic wounds, while around half of these 983,000 total patients received treatment [1]. As chronic wounds remain unhealed for three months or more, additional treatment beyond standard care wound may be required. In response to this growing clinical need, advanced wound healing technologies are being developed to heal refractory venous leg, diabetic foot, and pressure ulcers.

Advanced biophysical wound care therapies can apply mechanical, electrical, or light energy to re-stimulate the dysfunctional endogenous healing pathways in chronic wounds. Despite growing evidence of the clinical efficacy of these therapies, missing knowledge about the dose-response relationships prevents individually optimized treatment. Optimal treatment stimulation parameters remain unknown. Moreover, the lack of treatment protocols likely prevents wider clinical adoption of potentially beneficial therapies for wound healing.

This paper suggests a threefold approach to improve the clinical situation:

1. A systematic study on mixtures of biophysical modalities will be conducted, as previous biophysical wound care therapies focus on a single physical modality (e.g. mechanical or electrical or light radiation stimulation).

2. Experiments will be conducted on in vitro human 3D organotypic wound models. This design enables rapid evaluation including: quantification of the cellular responses to stimulation, repeatable experiments under various systematically prescribed stimulation patterns, and completion of a sufficient number of trial runs to obtain statistically significant results.

3. A system engineering approach will be applied to wound healing to formulate wound healing therapy as a control problem. A transfer function will be estimated and model equations derived and used for optimal model based control.

For successful development of a closed-loop control system, a number of research objectives need to be addressed:

- Determine the relationship between biophysical energies and the wound healing system response.

- Define the biophysical components of the response (e.g. investigate cellular responses and identify the highly correlated technical measurements for use in monitoring and predicting the cellular responses).

- Associate the mechanisms of action for the development of a composite therapeutic device and protocol for wound healing.

\subsection{Biophysical Therapies}

Advanced biophysical wound healing systems apply mechanical, electrical, or light energy to the wound bed. For example, negative pressure wound therapy (NPWT) applies vacuum pressure to a filled and sealed wound bed, while also removing tissue exudates. Electrical stimulation therapy (EST) applies imperceptible electrical fields across the wound tissue, while photobiomodulation (PBM) or low-level light therapy (LLLT) applies blue to near infrared light. Such biophysical-tissue interactions have been shown to re-stimulate the natural 
healing pathways.

Detailed knowledge of the pathways stimulated by these treatment modalities is growing. NWPT provides fluid removal, yet also derives efficacy from macroand micro-deformation, the latter being transferred to single cells by the extra cellular matrix (ECM) and potentially the integrin-mediated interaction of the cell with the matrix [2]. EST seems to act by enhanced recruitment of mast cells to the wound that may be mediated by so far poorly understood pathways of electrotaxis [3]. Finally, PBM seems to be acting at least in part through mitochondrial pathways and the generation of reactive oxygen species, the generation of ATP, and the stimulation of transcription factors [4].

\subsection{Stimulation Parameters}

Importantly, the dose-dependent tissue response to biophysical stimulation can be used to regulate healing pathways. For example, variation in NP magnitude and treatment waveform impact tissue perfusion and tissue granulation formation [5]. Variations in light energy show a dose-dependent response of cellular activation and inhibition at a threshold of $5 \mathrm{~J} / \mathrm{cm}^{2}$ [6]-[12]. Finally, variations in applied voltages [13] and stimulation duration [14] demonstrate a dose-dependent induced cell migration of keratinocytes and fibroblasts [15], respectively.

Generally, variation in the application of biophysical wound healing therapies include: device equipment and configuration, the magnitude and waveform of the biophysical energy, and treatment duration. Previous studies that have examined the impact of variable stimulation parameters on the tissue response are summarized by [16]. Tables 1-3 summarize the main relevant parameters per modality and provide main references to experimental evaluations.

Advanced biophysical wound care therapies are applied to unhealed wounds

Table 1. Relevant parameters for mechanical stimulation, adapted from [16].

\begin{tabular}{ccr}
\hline & Negative Pressure Wound Therapy (NPWT) \\
\hline Parameter & Relevance & Evidence \\
\hline Equipment & NPWT or Redon drainage & NPWT [17] \\
NP magnitude & Tissue perfusion modulation & $-50 \mathrm{mmHg}[18][19][20][21]$ to \\
NP waveform & Constant, square, or triangular & Inconclusive [26] [27]
\end{tabular}

Table 2. Relevant parameters for electrical stimulation, adapted from [16].

\begin{tabular}{|c|c|c|}
\hline \multicolumn{3}{|c|}{ High Voltage Pulsed Current (HVPC) } \\
\hline Parameter & Relevance & Evidence \\
\hline Waveform & Healing rates & $\begin{array}{c}\text { HVPC [13] [14] [15] } \\
\text { [28] [29] [30] }\end{array}$ \\
\hline Electrode configuration & In wound or around & $\begin{array}{c}\text { Electrode in wound } \\
{[31][32][33]}\end{array}$ \\
\hline Stimulation parameters & Optimisation & - \\
\hline Treatment duration & Wound closure time & $60+$ minutes $[28]$ \\
\hline
\end{tabular}


Table 3. Relevant parameters for light stimulation, adapted from [16].

\begin{tabular}{ccc}
\hline & Photobiomodulation & \\
\hline Parameter & Relevance & Evidence \\
\hline Equipment & LASER or LED & Either [6] [34] [35] \\
Wavelength & Blue to near infrared & Green [36] [37] [38] [39] [40] \\
Dose & Activating or inhibitory & $5 \mathrm{~J} / \mathrm{cm}^{2}$ threshold [6]-[12] [41] \\
Treatment duration & & $10-$ minutes [42] \\
\hline
\end{tabular}

to control and stabilize dysfunctions of the endogenous wound system for restimulation and regulation of the wound healing process for wound closure. Variations in the applications of mechanical, electrical, and light energies provide dose-dependent responses which can be used to regulate the healing pathways. This paper discusses the benefits of a closed-loop control system design to be used in research to characterize the wound healing system response to biophysical energies; the mechanical, light, and electrical components of the response; and the association of these mechanisms to be determined and related for the development of a composite therapeutic device and protocol for wound healing.

\section{Methods}

\subsection{Materials and Methods}

An in vitro 3D human cell model will be used to model the wound system. The 3D cell cultures are placed in 6-well plates to be experimentally treated in a parallel and reproducible manner. A machined dome adapter connects the culture plates to the stimulation devices. The adapter allows simultaneous stimulation and equal transmission of the same pressure wave form to each cell culture.

A negative pressure of prescribed waveform will be created by a NPWT device to apply exogenous mechanical energy into the cell assembly. Light energy of prescribed wavelength and dosage will be applied to the system from LEDs mounted on the ceiling of the assembly dome. Direct radiation without optical fibers is used in the first experiment series. Electrical energy will be applied into the tissue with thin needle electrodes fixed to a stabilizing lattice placed over the cell assembly.

A multidimensional controller sets the parameter space and shapes energy inputs applied into the wound system. Generally, treatment duration (time), signal intensity (energy magnitude), signal form (energy waveform), can be varied. Based on literature research of studies of the impact of variable stimulations parameters on wound healing outcomes [16], the variable input parameters are provided in Tables 1-3.

Wound area reduction and time to closure are standard outcome measurements that are easily obtained by video documentation. In addition, we will use electrical impedance (tomography) with a ring of electrodes around the wound for real time monitoring and staging of the system. Elaborated and extensive bi- 
ochemical and fluorescence based optical measurements at discrete points in time can be exploited to monitor cell growth and proliferation.

Thus, advanced biophysical wound therapy devices combined with self-constructed stimulation devices will be used to input mechanical, electrical, and light energy in parallel and in prescribed proportions into the wound system. Each available system has a selection of device equipment, stimulation parameters, and treatment duration. There are no standard therapy protocols yet. But, reviews on the variable methodologies used in biophysical therapies can offer some guidelines and ranges [16].

\subsection{Analysis}

The study design targets a closed-loop control system consisting of a controller, wound system, and negative feedback. The controller shapes the mechanical, electrical, and light energy by changing the parameter settings (Tables 1-3), whereby the prescribed parameter settings define the therapy to be transferred into the wound system. The wound system is the biological wound healing process itself, a complex tissue repair system, composed of its own endogenous control mechanisms. Negative feedback serves as a regulator or control to maintain wound healing system stability. Thus, the wound healing control system involves complex processes at the molecular, cellular, and tissue levels, which are interconnected and regulated by endogenous and exogenous tissue responses to biophysical energies that are not yet well understood.

For the purpose of control, we will pursue two paths. First, to consider the wound system as a black box with certain properties (e.g.: piecewise linear, time delays etc.). Secondly, trying to capture the detailed wound characteristics using a model based approach. Then, creating a model-based controller may account for the expected nonlinear phenomena of the complex biological system.

We expect classical control methods via identification of transfer functions, also (physiologic) model based control will be an option for the final therapeutic system. Some first wound healing control models [43] will serve as a starting point. Models will be implemented and evaluated for their predictive properties. Models will be proven, tested, and simplified until prediction accuracy is significantly reduced. Robustness of the control system will be determined.

Due to the Arndst-Schultz Law, which describes the biphasic dose-dependent effects of PBM, we expect highly nonlinear system behaviour. Similarly, we expect the interactions of mechanical and electrical modalities to be nonlinear. Therefore, in a number of experiments, the relevant parameters space will be investigated to establish a mathematical model description which will finally simplify the design of the overall control system.

\section{Results}

We propose a closed-loop control wound system to be used in determining the impact of variation in biophysical energy inputs on wound outcome measures. The wound system will be monitored and measured by sensors to evaluate the 
progress of wound healing. This design of a closed-loop control system using multiple and concurrent biophysical wound healing therapies will be used to determine the tissue response to prescribed input parameters.

Main results of this paper include the description of the design of the wound healing therapy control system, defined constraints of the ranges of the biophysical energies to be applied, and design of a system to provide concurrent input energies using multiple biophysical therapy modalities. Given an analysis of the literature [16], a parameter space is defined. To better understand the complex reaction of wound cells to stimulation we need to explore the parameter space. Thus, we apply a preliminary constraint on the range of the 9 dimensional parameter space as provided in Table 4.

We selected NP magnitudes ranging from -30 to $-80 \mathrm{mmHg}$, as previous studies have shown similar tissue perfusion responses at -50 [18] [19] [20] [21] and $-80 \mathrm{mmHg}$ [22] [23] [24] [25], as compared to the initial uses of $-120 \mathrm{mmHg}$. Sinusoidal and square wave NP waveforms are selected and continuous waveforms are not studied, as we suspect that changes in the applied waveform modulate the biological response of granulation tissue formation [26]. Treatment duration will be ongoing until wound closure, and periodic wound assessment measurements will be made.

We continue to apply PBM from blue to near infrared wavelengths. Although red wavelengths are almost exclusively used, studies applying either blue, green, or red light have shown more consistent results with the use of green wavelengths [16]. Selected PBM dose ranges are from 2 to $10 \mathrm{~J} / \mathrm{cm}^{2}$, which are similar to ranges used in studies showing the cellular dose-dependent activation and inhibition threshold [7] [8] [9] [10]. Treatment durations will be less than $10 \mathrm{mi}$ nutes [42].

Finally, a high voltage pulsed current (HVPC) is selected, as suggested in the literature [44] [45]. Electrical stimulation parameters have not been studied for optimal performance ranges, so we will explore the effects of various currents and voltages. Treatment duration of at least 60 minutes seems to activate cellular

Table 4. Stimulation parameters and experimental ranges

\begin{tabular}{ccc}
\hline \multicolumn{2}{c}{ Potential stimulation parameters and experimental ranges } \\
\hline Device & Parameter & Constraint \\
\hline $\begin{array}{c}\text { Negative pressure wound } \\
\text { therapy }\end{array}$ & NP amplitude & -30 to $-80 \mathrm{mmHg}$ \\
& Treatment duration & Sinusoidal and square \\
Electrical stimulation & Waveform & Minutes to days \\
& Stimulation parameters & High voltage pulsed current \\
& Treatment duration & Current, voltage \\
& Wavelength & $60+$ minutes \\
Photobiomodulation & Dose & Blue to near infrared \\
& Treatment duration & 2 to $10 \mathrm{~J} / \mathrm{cm}^{2}$ \\
& & 10 -minutes
\end{tabular}


pathways [28].

\section{Discussion}

Regulation and control of the wound healing system seems possible, if sufficient knowledge about biophysical-tissue interactions can be experimentally acquired and the system behaviour response can be uniquely described. This work presents an experimental device that is specified and drafted to input differently shaped mechanical, electrical, and light energies into an in vitro 3D cell wound model. We aim to characterize the wound healing system response to biophysical energies; the mechanical, light, and electrical components of the response; and the association of these mechanisms to be determined and related for the development of a composite therapeutic device and protocol for wound healing. The currently unknown optimal stimulation parameters could be subsequently derived and errors in the feedback of the system could show where therapies can be introduced.

To date, the optimal treatment parameters for individualized wound healing needs remain unknown. Moreover diverse approaches in equipment development, scientific research, and clinical application continue without a defined systems engineering basis for explanation of effects. Our design introduces a wound healing control system that provides a systematic exploration of the biophysical therapy device stimulation parameter space. This work also creates a foundation for automated control of the wound healing process.

Advantages of a systems approach for wound healing are that established methods are applicable even if partial knowledge in the domain is available with direct link to optimal control of systems. A control approach can build on a rich variety of algorithms with demonstrated properties such as accuracy, robustness, and response time. Knowing form and function characteristics associated with endogenous healing processes can guide controller design and therefore will have positive influences on therapeutic device design.

Advantages of an in vitro model are that molecular, cellular, and tissue levels responses are quantifiable by standard chemical, biological, and optical measurement techniques. Disadvantages of an in vitro model are that pre-clinical findings may not transfer accurately to the clinical setting. However, in vitro laboratory studies provide better access to the biological processes i.e. complex measurements of wound healing processes become available. We will be able to quantify and evaluate the pre-clinical evidence of the biophysical-tissue response patterns, cellular proliferation, and migration, and time to wound closure.

\section{Conclusion}

A control system design for technically assisted wound healing is introduced that may offer individualized optimal wound healing therapy in the future.

\section{References}

[1] Heyer, K., et al. (2016) Epidemiology of Chronic Wounds in Germany: Analysis of 
statutory health insurance data. Wound Repair Regen, 24 (2), 434-442. https://doi.org/10.1111/wrr.12387

[2] Huang, C., et al. (2014) Effect of Negative Pressure Wound Therapy on Wound Healing. CurrProblSurg, 51, 301-31. https://doi.org/10.1067/j.cpsurg.2014.04.001

[3] Torkaman, G. (2014) Electrical Stimulation of Wound Healing: A Review of Animal Experimental Evidence. Advances in Wound Care, 3, 202-218. https://doi.org/10.1089/wound.2012.0409

[4] Chung, H., et al. (2012) TheNuts and Bolts of Low-Level Laser (Light) Therapy. Ann Biomed Eng, 40, 516-33. https://doi.org/10.1007/s10439-011-0454-7

[5] Glass, G.E. and Nanchahal, J. (2012) The Methodology of Negative Pressure Wound Therapy: Separating Fact From Fiction. J PlastReconstrAesthetSurg, 65, 989-1001. https://doi.org/10.1016/j.bjps.2011.12.012

[6] Chaves, M.E., et al. (2014) Effects of Low-Power Light Therapy on Wound Healing: LASER x LED. An Bras Dermatol, 89, 616-23.

[7] Hawkins, D. and Abrahamse, H. (2005) Biological Effects of Helium-Neon Laser Irradiation on Normal and Wounded Human Skin Fibroblasts. Photomed Laser Surg, 23, 251-9. https://doi.org/10.1089/pho.2005.23.251

[8] Hawkins, D. and Abrahamse, H. (2006) Effect of Multiple Exposures of Low-Level Laser Therapy on the Cellular Responses of Wounded Human Skin Fibroblasts. Photomed Laser Surg, 24, 705-14. https://doi.org/10.1089/pho.2006.24.705

[9] Houreld, N. and Abrahamse, H. (2007) In Vitro Exposure of Wounded Diabetic Fibroblast Cells to a Helium-Neon Laser at 5 and $16 \mathrm{~J} / \mathrm{cm} 2$. Photomed Laser Surg, 25, 78-84.https://doi.org/10.1089/pho.2006.990

[10] Houreld, N.N. and Abrahamse, H. (2008) Laser Light Influences Cellular Viability and Proliferation in Diabetic-Wounded Fibroblast Cells in a Dose- and Wavelength-Dependent Manner. Lasers Med Sci, 23, 11-8. https://doi.org/10.1007/s10103-007-0445-y

[11] Huang, Y.Y., et al. (2009) Biphasic Dose Response in Low Level Light Therapy. Dose Response, 7, 358-83. https://doi.org/10.2203/dose-response.09-027.Hamblin

[12] Huang, Y.Y., et al. (2011) Biphasic Dose Response in Low Level Light Therapy - An Update. Dose Response, 9, 602-18.

[13] Nishimura, K.Y., et al. (1996) Human Keratinocytes Migrate to the Negative Pole in Direct Current Electric Fields Comparable to Those Measured in Mammalian Wounds. Journal of Cell Science, 109, 199-207.

[14] Bikson, M., et al. (2009) Establishing Safety Limits for Transcranial Direct Current Stimulation. ClinNeurophysiol, 120, 1033-4.

[15] Guo, A., et al. (2010) Effects of Physiological Electric Fields on Migration of Human Dermal Fibroblasts. J Invest Dermatol, 130, 2320-7.

[16] Parente, J.D., et al. (2016) Methodologies of Biophysical Wound Healing Therapies. Journal of Biomedical Science and Engineering, 9, 171. https://doi.org/10.4236/jbise.2016.910b022

[17] Wild, T., et al. (2008) Definition of Efficiency in Vacuum Therapy--A Randomised Controlled Trial Comparing with V.A.C. Therapy. Int Wound J, 5, 641-7.

[18] Isago, T., et al. (2003) Effects of Different Negative Pressures on Reduction of Wounds in Negative Pressure Dressings. J Dermatol, 30, 596-601.

[19] Lindstedt, S., et al. (2008) Impact of Different Topical Negative Pressure Levels on Myocardial Microvascular Blood Flow. CardiovascRevasc Med, 9, 29-35.

[20] Lindstedt, S., et al. (2008) A Compare between Myocardial Topical Negative Pressure Levels of $-25 \mathrm{mmHg}$ and $-50 \mathrm{mmHg}$ in a Porcine Model. BMC CardiovascDisord, 8,14 .

[21] Malmsjo, M., et al. (2009) Negative-pressure Wound Therapy Using Gauze or Open-Cell Polyurethane Foam: Similar Early Effects on Pressure Transduction and Tissue Contraction in an Experimental Porcine Wound Model. Wound Repair Re- 
gen, 17, 200-205.

[22] Borgquist, O., et al. (2009) Tissue Ingrowth into Foam but Not into Gauze during Negative Pressure Wound Therapy. Wounds, 21, 302-309.

[23] Borgquist, O., et al. (2010) Micro- andMacromechanical Effects on the Wound Bed of Negative Pressure Wound Therapy Using Gauze and Foam. Ann PlastSurg, 64, 789-793.

[24] [24] Borgquist, O., et al. (2010) Wound Edge Microvascular Blood Flow during Negative-Pressure Wound Therapy: Examining the Effects of Pressures from -10 to -175 mmHg. PlastReconstrSurg, 125, 502-509.

[25] Borgquist, O., et al. (2011) The Influence of Low and High Pressure Levels during Negative-Pressure Wound Therapy on Wound Contraction and Fluid Evacuation. PlastReconstrSurg, 127, 551-9. https://doi.org/10.1097/PRS.0b013e3181fed52a

[26] Dastouri, P., et al. (2011) Waveform Modulation of Negative-Pressure Wound Therapy in the Murine Model. PlastReconstrSurg, 127, 1460-1466.

[27] Morykwas, M.J., et al. (1997) Vacuum-assisted Closure: A New Method for Wound Control and Treatment: Animal Studies and Basic Foundation. Ann PlastSurg, 38, 553-562.https://doi.org/10.1097/00000637-199706000-00001

[28] Ahmad, E.T. (2008) High-voltage Pulsed Galvanic Stimulation: Effect of Treatment Duration on Healing of Chronic Pressure Ulcers. Ann Burns Fire Disasters, 21, 124-128.

[29] Stefanovska, A., et al. (1993) Treatment of Chronic Wounds by Means of Electric and Electromagnetic Fields. Part 2. Value of FES parameters for pressure sore treatment. Med BiolEngComput, 31, 213-220.https://doi.org/10.1007/bf02458039

[30] Baker, L.L., et al. (1996) Effect of Electrical Stimulation Waveform on Healing of Ulcers in Human Beings with Spinal Cord Injury. Wound Repair Regen, 4, 21-8.

[31] Barker, A.T., et al.(1982) TheGlabrous Epidermis of Cavies Contains a Powerful Battery. Am J Physiol, 242, R358-66.

[32] Karba, R., et al. (1997) DC Electrical Stimulation for Chronic Wound Healing Enhancement Part 1. Clinical Study and Determination of Electrical Field Distribution in the Numerical Wound Model. Bioelectrochemistry and bioenergetics, 43, 265-270.https://doi.org/10.1016/S0302-4598(96)05192-6

[33] Semrov, D., et al. (1997) DC Electrical Stimulation for Chronic Wound Healing Enhancement. Part 2. Parameter determination by numerical modelling. Bioelectrochemistry and bioenergetics, 43, 271-278. https://doi.org/10.1016/S0302-4598(96)05193-8

[34] Enwemeka, C. S. (2006) The place of coherence in light induced tissue repair and pain modulation. Photomed Laser Surg, 24, 457.

https://doi.org/10.1089/pho.2006.24.457

[35] Karu, T. (1987) Photobiological fundamentals of low-power laser therapy. IEEE Journal of Quantum Electronics, 23, 1703-1717. https://doi.org/10.1109/JQE.1987.1073236

[36] Adamskaya, N., et al. (2011) Light therapy by blue LED improves wound healing in an excision model in rats. Injury, 42, 917-21. https://doi.org/10.1016/j.injury.2010.03.023

[37] Cheon, M.W., et al.(2013) Low Level Light Therapy by Red-Green-Blue LEDs Improves Healing in an Excision Model of Sprague-Dawley rats. Personal and Ubiquitous Computing, 17, 1421-1428.https://doi.org/10.1007/s00779-012-0577-3

[38] De Sousa, A.P.C., et al. (2013) Laser and LED Phototherapies on Angiogenesis. Lasers in Medical Science, 28, 981-987.https://doi.org/10.1007/s10103-012-1187-z

[39] De Sousa, A.P.C., et al. (2010) Effect of LED Phototherapy of Three Distinct Wavelengths on Fibroblasts on Wound Healing: A Histological Study in a Rodent Model. Photomedicine and laser surgery, 28, 547-552. https://doi.org/10.1089/pho.2009.2605 
[40] Fushimi, T., et al. (2012) Green Light Emitting Diodes Accelerate Wound Healing: Characterization of the Effect and Its Molecular Basis in Vitro and in Vivo. Wound Repair Regen, 20, 226-35. https://doi.org/10.1111/j.1524-475X.2012.00771.x

[41] Medrado, A.R., et al. (2003) Influence of Low Level Laser Therapy on Wound Healing and Its Biological Action upon Myofibroblasts. Lasers Surg Med, 32, 239-44. https://doi.org/10.1002/lsm.10126

[42] Lanzafame, R.J., et al.(2007) Reciprocity of Exposure Time and Irradiance on Energy Density during Photoradiation on Wound Healing in a Murine Pressure Ulcer Model. Lasers Surg Med, 39, 534-542. https://doi.org/10.1002/1sm.20519

[43] O'Clock, G.D. (2014) A multi-scale Feedback Control System Model for Wound Healing Electrical Activity: Therapeutic Device/Protocol Implications. Conf Proc IEEE Eng Med BiolSoc, 2014, 3021-5. https://doi.org/10.1109/embc.2014.6944259

[44] Kawasaki, L., et al. (2014) The Mechanisms and Evidence of Efficacy of Electrical Stimulation for Healing of Pressure Ulcer: A Systematic Review. Wound Repair Regen, 22, 161-173. https://doi.org/10.1111/wrr.12134

[45] Polak, A., et al. (2014) High-Voltage Pulsed Current Electrical Stimulation in Wound Treatment. Adv Wound Care (New Rochelle), 3, 104-117.

Submit or recommend next manuscript to SCIRP and we will provide best service for you:

Accepting pre-submission inquiries through Email, Facebook, LinkedIn, Twitter, etc. A wide selection of journals (inclusive of 9 subjects, more than 200 journals)

Providing 24-hour high-quality service

User-friendly online submission system

Fair and swift peer-review system

Efficient typesetting and proofreading procedure

Display of the result of downloads and visits, as well as the number of cited articles

Maximum dissemination of your research work

Submit your manuscript at: http://papersubmission.scirp.org/

Or contact jbise@scirp.org 